\title{
A refinement of the Shuffle Conjecture with cars of two sizes and $t=1 / q$
}

\author{
Angela Hicks* And Emily Leven ${ }^{\dagger}$
}

\begin{abstract}
The original Shuffle Conjecture of [12] has a symmetric function side and a combinatorial side. The symmetric function side may be simply expressed as $\left\langle\nabla e_{n}, h_{\mu}\right\rangle$ where $\nabla$ is the Macdonald polynomial eigen-operator of [3] and $h_{\mu}$ is the homogeneous basis indexed by $\mu=\left(\mu_{1}, \mu_{2}, \ldots, \mu_{k}\right) \vdash n$. The combinatorial side $q$, $t$-enumerates a family of Parking Functions whose reading word is a shuffle of $k$ successive segments of $123 \ldots n$ of respective lengths $\mu_{1}, \mu_{2}, \ldots, \mu_{k}$. It can be shown that for $t=1 / q$ the symmetric function side reduces to a product of $q$-binomial coefficients and powers of $q$. This reduction suggests a surprising combinatorial refinement of the general Shuffle Conjecture. Here we prove this refinement for $k=2$ and $t=1 / q$. The resulting formula gives a $q$-analogue of the well-studied Narayana numbers.
\end{abstract}

AMS 2000 SUbJeCt Classifications: Primary 05E05; secondary 05E10. KeYwords AND PHRASEs: Parking functions, shuffle Conjecture, dinv, Narayana numbers.

\section{Introduction}

A Dyck path in the $n \times n$ lattice square starts at the southwest corner of the square and proceeds to the northeast corner with $n$ north edges and $n$ east edges, always remaining weakly above the diagonal joining these the same two corners. Here and after, we will refer to the cells crossed by this diagonal as in the main diagonal of the square.

Here we visualize a Parking Function as a Dyck path in the $n \times n$ lattice square whose north steps are labeled in a column increasing way by the integers $\{1,2, \ldots, n\}$. For convenience, we place the label of a north edge in the cell immediately to the east of that edge. This visual representation has its origins in [7], where it is used as a geometric way of depicting preference

arXiv: 1304.7026

*Supported by NSF grant DMS-1303761.

†Supported by NSF grant DGE-1144086. 
functions that park the cars on a one way street (see [13]). We will also borrow the term cars for the labels of the north edges.

For computational convenience, Parking Functions may also be represented as two-line arrays:

$$
\mathrm{PF}=\left[\begin{array}{llll}
v_{1} & v_{2} & \ldots & v_{n} \\
u_{1} & u_{2} & \ldots & u_{n}
\end{array}\right]
$$

with $u_{1}, u_{2}, \ldots, u_{n}$ integers satisfying

$$
u_{1}=0 \quad \text { and } \quad 0 \leq u_{i} \leq u_{i-1}+1
$$

and $V=\left(v_{1}, v_{2}, \ldots, v_{n}\right)$ a permutation in $S_{n}$ satisfying $u_{i}=u_{i-1}+1 \Rightarrow v_{i}>$ $v_{i-1}$. The component $u_{i}$ represents the number of full cells in the $i$ th row of the diagram that are east of the path and strictly west of the main diagonal. The component $v_{i}$ gives the car that resides in the $i^{\text {th }}$ row. An example of these two corresponding representations is given below.

$$
\left[\begin{array}{llllllll}
4 & 6 & 8 & 1 & 3 & 2 & 7 & 5 \\
0 & 1 & 2 & 2 & 3 & 0 & 1 & 1
\end{array}\right] \quad \Longleftrightarrow
$$

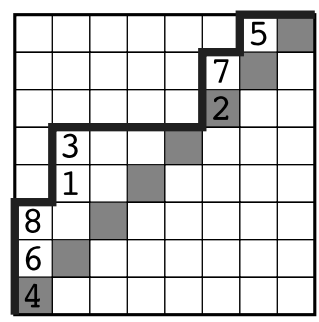

We will denote by $\sigma(\mathrm{PF})$ the permutation obtained by successive right to left readings of the components of the permutation $V=\left(v_{1}, v_{2}, \ldots, v_{n}\right)$ according to decreasing values of $u_{1}, u_{2}, \ldots, u_{n}$. We will call $\sigma(\mathrm{PF})$ the diagonal word of PF. This given, each Parking Function is assigned two statistics

$$
\operatorname{area}(\mathrm{PF})=\sum_{i=1}^{n} u_{i}
$$

and

$$
\begin{aligned}
\operatorname{dinv}(\mathrm{PF})= & \sum_{1 \leq i<j \leq n} \chi\left(u_{i}=u_{j} \& v_{i}<v_{j}\right) \\
& +\sum_{1 \leq i<j \leq n} \chi\left(u_{i}=u_{j}+1 \& v_{i}>v_{j}\right) .
\end{aligned}
$$


It is easily seen that area $(\mathrm{PF})$ gives the total number of cells between the supporting Dyck path and the main diagonal. The notation $\operatorname{dinv}(\mathrm{PF})$ is an abbreviation of the term "diagonal inversions." Note that two cars in the same diagonal with the car on the left smaller than the car on the right will contribute a unit to $\operatorname{dinv}(\mathrm{PF})$ called a primary dinv. Likewise, a car on the left that is bigger than a car on the right with the latter in the adjacent lower diagonal contributes a unit to $\operatorname{dinv}(\mathrm{PF})$ called a secondary dinv. In the example above, $\sigma(\mathrm{PF})=31857624$, area $(\mathrm{PF})=10$ and $\operatorname{dinv}(\mathrm{PF})=4$.

For two integers $a+b=n$, let us refer to $1,2, \ldots, a$ as the small cars and $a+1, a+2, \ldots, n$ as the $b i g$ cars and let $\mathcal{P F}_{a, b}^{(r, s)}$ denote the collection of Parking Functions whose reading word is a shuffle of the two words $12 \ldots a$ and $a+1 a+2 \ldots n$ and which have $r$ small cars and $s$ big cars in the main diagonal. Similarly, let $\mathcal{P} \mathcal{F}_{a, b}^{(s)}=\cup_{r=0}^{a} \mathcal{P} \mathcal{F}_{a, b}^{(r, s)}$ denote the collection of Parking Functions whose reading word is a shuffle of the two words $12 \ldots a$ and $a+1 a+2 \ldots n$ and which have $s$ big cars in the main diagonal, and let $\mathcal{P} \mathcal{F}_{a, b}=\cup_{r=0}^{a} \cup_{s=0}^{b} \mathcal{P} \mathcal{F}_{a, b}^{(r, s)}$.

Our main result here can be stated as follows

Theorem 1.1. Let $0 \leq s \leq b$ and $0 \leq r \leq a$. If $s<b$, then

$$
\begin{aligned}
& \sum_{\operatorname{PF} \in \mathcal{P F}_{a, b}^{(r, s)}} q^{\operatorname{coarea}(\mathrm{PF})+\operatorname{dinv}(\mathrm{PF})} \\
& =q^{\left(\begin{array}{c}
a+b \\
2
\end{array}\right)-(a-r+1)(b-s)}\left[\begin{array}{c}
a+b-s-1 \\
a
\end{array}\right]_{q}\left[\begin{array}{c}
a-r+b-s \\
a-r
\end{array}\right]_{q} \\
& \quad \times\left[\begin{array}{c}
r+s \\
s
\end{array}\right]_{q} \frac{[r]_{q}}{[a-r+b-s]_{q}},
\end{aligned}
$$

and if $s=b$, then

$$
\sum_{\mathrm{PF} \in \mathcal{P F}_{a, b}^{(r, b)}} q^{\operatorname{coarea}(\mathrm{PF})+\operatorname{dinv}(\mathrm{PF})}=\chi(a=r) q^{\left(\begin{array}{c}
a+b \\
2
\end{array}\right)}\left[\begin{array}{c}
a+b \\
a
\end{array}\right]_{q}
$$

where coarea $(\mathrm{PF})=\left(\begin{array}{l}n \\ 2\end{array}\right)-\operatorname{area}(\mathrm{PF})$.

To see why this constitutes a refinement of the $t=1 / q$ and $k=2$ case of the Shuffle Conjecture we need to review some background. A more thorough introduction to the relevant tools in symmetric function theory can be found in section 2. To begin, the Shuffle Conjecture of [12] can be stated as follows. 
Conjecture 1.1. For $\mu=\left(\mu_{1}, \mu_{2}, \ldots, \mu_{k}\right) \vdash n$ we have

$$
\left\langle\nabla e_{n}, h_{\mu}\right\rangle=\sum_{\mathrm{PF} \in \mathcal{P} \mathcal{F}_{n}} t^{\operatorname{area}(\mathrm{PF})} q^{\operatorname{dinv}(\mathrm{PF})} \chi\left(\sigma(\mathrm{PF}) \in E_{1} \sqcup E_{2} \sqcup \cdots \sqcup E_{k}\right)
$$

where " $\mathcal{P} \mathcal{F}_{n}$ " denotes the collection of Parking Functions in the $n \times n$ lattice square, $E_{1}, E_{2}, \ldots, E_{k}$ are successive segments of the word $123 \ldots n$ of respective lengths $\mu_{1}, \mu_{2}, \ldots, \mu_{k}$ and "Ш" denotes shuffling.

Now it was already noticed by [7] that

$$
\left.q^{\left(\begin{array}{c}
n \\
2
\end{array}\right)} \nabla e_{n}\right|_{t=1 / q}=\frac{1}{[n+1]_{q}} e_{n}\left[X[n+1]_{q}\right]
$$

Using this identity, it is not difficult to derive that Conjecture 1.1 implies the following, also open, conjecture. For an explicit computation of this in the two shuffle case, see [1].

\section{Conjecture 1.2.}

$$
\begin{aligned}
& \sum_{\mathrm{PF} \in \mathcal{P} \mathcal{F}_{n}} q^{\text {coarea }(\mathrm{PF})+\operatorname{dinv}(\mathrm{PF})} \chi\left(\sigma(\mathrm{PF}) \in E_{1} \amalg E_{2} \amalg \cdots \uplus E_{k}\right) \\
& =\frac{1}{[n+1]_{q}} \prod_{i=1}^{k} q^{\left(\begin{array}{c}
\mu_{i} \\
2
\end{array}\right)}\left[\begin{array}{c}
n+1 \\
\mu_{i}
\end{array}\right]_{q} .
\end{aligned}
$$

The two parameters $s$ and $r$ in Theorem 1.1 induce a partition of studied parkings functions. One can sum the generating functions of each part of this partition to obtain a proof for the case $\mathrm{k}=2$ of Conjecture 1.2. More precisely,

Theorem 1.2. For $a \geq 0$ and $b \geq 0$,

$$
\sum_{\mathrm{PF} \in \mathcal{P F}_{a, b}} q^{\operatorname{coarea}(\mathrm{PF})+\operatorname{dinv}(\mathrm{PF})}=\frac{q^{\left(\begin{array}{c}
a \\
2
\end{array}\right)+\left(\begin{array}{l}
b \\
2
\end{array}\right)}}{[a+b+1]_{q}}\left[\begin{array}{c}
a+b+1 \\
a
\end{array}\right]_{q}\left[\begin{array}{c}
a+b+1 \\
b
\end{array}\right]_{q} .
$$

Our proofs of Theorems 1.1 and 1.2 are based on two key recursions which may be stated as follows. For parameters $a, b, r, s$, let us set

$$
\operatorname{Parkq}_{a, b}^{(r, s)}(q)=\sum_{\mathrm{PF} \in \mathcal{P F}_{a, b}^{(r, s)}} q^{\operatorname{coarea}(\mathrm{PF})+\operatorname{dinv}(\mathrm{PF})}
$$


and

$$
\operatorname{Parkq}_{a, b}^{(s)}(q)=\sum_{\mathrm{PF} \in \mathcal{P F}_{a, b}^{(s)}} q^{\operatorname{coarea}(\mathrm{PF})+\operatorname{dinv}(\mathrm{PF})}
$$

Similarly, let

$$
\operatorname{Parkqt}_{a, b}^{(r, s)}(q, t)=\sum_{\mathrm{PF} \in \mathcal{P F}_{a, b}^{(r, s)}} t^{\operatorname{area}(\mathrm{PF})} q^{\operatorname{dinv}(\mathrm{PF})}
$$

and

$$
\operatorname{Parkqt}_{a, b}^{(s)}(q, t)=\sum_{\mathrm{PF} \in \mathcal{P F}_{a, b}^{(s)}} t^{\operatorname{area}(\mathrm{PF})} q^{\operatorname{dinv}(\mathrm{PF})}
$$

This given, we have the following recursions.

Proposition 1.1. If $0 \leq r \leq a$ and $0 \leq s<b$, then

$$
\begin{aligned}
\operatorname{Parkq}_{a, b}^{(r, s)}(q)= & q^{(s+r)(a+b)-\left(\begin{array}{c}
s+r+1 \\
2
\end{array}\right)-1}\left[\begin{array}{c}
s+r \\
s
\end{array}\right]_{q} \\
& \times \sum_{h=1}^{b-s}\left[\begin{array}{c}
r+h-1 \\
h
\end{array}\right]_{q} \sum_{k=0}^{a-r} \operatorname{Parkq}_{a-r, b-s-1}^{(k, h-1)}(q) \\
= & q^{(s+r)(a+b)-\left(\begin{array}{c}
(s+r+1 \\
2
\end{array}\right)-1}\left[\begin{array}{c}
s+r \\
s
\end{array}\right]_{q} \\
& \times \sum_{h=1}^{b-s}\left[\begin{array}{c}
r+h-1 \\
h
\end{array}\right]_{q} \operatorname{Parkq}_{a-r, b-s-1}^{(h-1)}(q) .
\end{aligned}
$$

Proposition 1.2. If $1 \leq a$ and $0 \leq s \leq b$, then

(1.11) $\operatorname{Parkq}_{a, b}^{(s)}(q)=q^{\left(\begin{array}{c}a+b \\ 2\end{array}\right)-(b-s)-\left(\begin{array}{c}a+b-s-1 \\ 2\end{array}\right)} \sum_{r=1}^{a}\left[\begin{array}{c}s+r \\ r\end{array}\right]_{q} \operatorname{Parkq}_{b-s, a-1}^{(r-1)}(q)$

The identity in Proposition 1.1 is the $t=1 / q$ specialization of an identity for Parkqt $t_{a, b}^{(r, s)}$ proved by the first author in [2] and Proposition 1.2 is a specialization of a recursion for Parkqt ${ }_{a, b}^{(s)}$ originally discovered by [11]. Below we reproduce a simple surjective proof of the latter which was given by the first author and Stout in [9]. 
In point of fact, [11] proved, by a highly non-trivial sequence of manipulations, that

$$
\operatorname{Parkqt}_{a, b+1}^{(s)}(q, t)=\left\langle\Delta_{h_{a}} E_{b+1, s}, e_{a+b+1}\right\rangle
$$

where $\Delta_{h_{a}}$ is one of a family of Macdonald polynomial eigen-operators constructed in [4] and the $E_{n, k}$ are the symmetric polynomials introduced by [6]. The simple form given here for $\operatorname{Parkq}_{a, b}^{(r, s)}(q)$ suggests that $\operatorname{Parkqt}_{a, b}^{(r, s)}(q, t)$, may also be expressible in terms of symmetric functions in a manner that refines (1.12). If this can be carried out for general multicar sizes it should yield a significant refinement of the classical Shuffle Conjecture. This task is certainly worth pursuing in future work.

\section{The symmetric function side}

For the sake of completion, we provide a brief survey of the symmetric function tools necessary to understand Conjectures 1.1 and 1.2. For a more thorough introduction see [14] and [8]. The space of symmetric polynomials will be denoted by $\Lambda$. The space of homogeneous symmetric polynomials of degree $n$ will be denotes by $\Lambda^{=n}$. We will express symmetric functions in terms of the following classic bases for $\Lambda^{=n}$ indexed by partitions of $n$ :

- the power basis $\left\{p_{\mu}\right\}_{\mu \vdash n}$

- the homogeneous basis $\left\{h_{\mu}\right\}_{\mu \vdash n}$

- the elementary basis $\left\{e_{\mu}\right\}_{\mu \vdash n}$

- the Schur basis $\left\{s_{\mu}\right\}_{\mu \vdash n}$

Below $\langle\cdot, \cdot\rangle$ denotes the usual scalar product on symmetric functions defined by

$$
\left\langle s_{\lambda}, s_{\mu}\right\rangle=\chi(\lambda=\mu) .
$$

For each partition $\lambda$, let $\lambda^{\prime}$ denote the conjugate partition. We will also make use of the involution $\omega$ defined by $\omega s_{\lambda}=s_{\lambda^{\prime}}$. Note that since $e_{k}=s_{\left(1^{k}\right)}$ and $h_{k}=s_{(k)}$, we have that $\omega h_{k}=e_{k}$ and, in general, $\omega h_{\lambda}=e_{\lambda}$.

If $E=E\left(t_{1}, t_{2}, \ldots\right)$ is a formal Laurent series in the variables $t_{1}, t_{2}, \ldots$, we define

$$
p_{k}[E]=E\left(t_{1}^{k}, t_{2}^{k}, \ldots\right) \text {. }
$$

More generally, if $F$ is any symmetric function, it can be expressed as a polynomial $F=Q\left(p_{1}, p_{2}, \ldots\right)$ in the power basis. This given, we define

$$
F[E]=Q\left(p_{1}[E], p_{2}[E], \ldots\right) .
$$




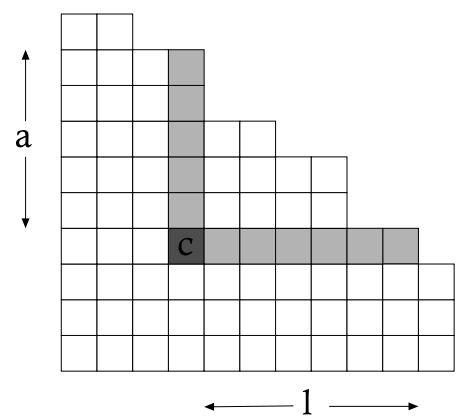

Figure 1: The Ferrers diagram of a partition.

This process is referred to as plethystic substitution.

It will be convenient to denote a partition by its (French) Ferrers diagram as in Figure 1. Given a partition $\mu$ and a cell $c \in \mu$, the parameters $a=a_{\mu}(c)$ and $l=l_{\mu}(c)$, called the arm and leg, give the number of cells north and east of $c$, respectively. Also define

$$
n(\mu)=\sum_{c \in \mu} l_{\mu}(c)=\sum_{i=1}^{l(\mu)}(i-1) \mu_{i} .
$$

Let

$$
w_{\mu}(q, t)=\prod_{c \in \mu}\left(q^{a_{\mu}(c)}-t^{l_{\mu}(c)+1}\right)\left(t^{l_{\mu}(c)}-q^{a_{\mu}(c)+1}\right)
$$

and

$$
T_{\mu}=t^{n(\mu)} q^{n\left(\mu^{\prime}\right)} .
$$

For any partition $\mu$, let $z_{\mu}$ be the order of the stabilizer of a permutation with cycle structure $\mu$. We have that

$$
\left\langle p_{\lambda}, p_{\mu}\right\rangle=z_{\mu} \chi(\lambda=\mu) .
$$

A related scalar product, called the star scalar product, is given by

$$
\left\langle p_{\lambda}, p_{\mu}\right\rangle_{*}=(-1)^{|\mu|-l(\mu)} z_{\mu} \chi(\lambda=\mu) \prod_{i}\left(1-t^{\mu_{i}}\right)\left(1-q^{\mu_{i}}\right) .
$$

This given, the modified Macdonald polynomials $\left\{\tilde{H}_{\mu}(X ; q, t)\right\}_{\mu}$ are the unique symmetric function basis which is upper-triangularly related to the 
Schur basis $\left\{s_{\mu}(x)\right\}_{\mu}$ with respect to $\langle\cdot, \cdot\rangle_{*}$ and satisfies the orthogonality condition

$$
\left\langle\tilde{H}_{\lambda}(X ; q, t), \tilde{H}_{\mu}(X ; q, t)\right\rangle_{*}=w_{\mu}(q, t) \chi(\lambda=\mu) .
$$

Following [3], we let $\nabla$ denote the eigen-operator for the Macdonald polynomials with eigenvalue $T_{\mu}$.

Now we have the tools to sketch a proof of the following lemma.

Lemma 2.1. Conjecture 1.1 implies Conjecture 1.2.

Proof. By (1.4), it is sufficient to show that

$$
\left\langle e_{n}\left[X[n+1]_{q}\right], h_{\mu_{1}} h_{\mu_{2}} \ldots h_{\mu_{k}}\right\rangle=\prod_{i=1}^{k} q^{\left(\begin{array}{c}
\mu_{i} \\
2
\end{array}\right)}\left[\begin{array}{c}
n+1 \\
\mu_{i}
\end{array}\right]_{q} .
$$

It is well known that for any $X$ and $Y$ we have

$$
h_{n}[X Y]=\sum_{\mu \vdash n} s_{\mu}[X] s_{\mu}[Y]
$$

This expression is known as the Cauchy identity. When $Y=[n+1]_{q}$, applying $\omega$ with respect to $X$ gives

$$
e_{n}\left[X[n+1]_{q}\right]=\sum_{\mu \vdash n} s_{\mu^{\prime}}[X] s_{\mu}\left[[n+1]_{q}\right]=\sum_{\mu \vdash n} s_{\mu}[X] s_{\mu^{\prime}}\left[[n+1]_{q}\right] .
$$

Hence by (2.1), for any partition $\lambda$ we have that

$$
\left\langle e_{n}\left[X[n+1]_{q}\right], s_{\lambda}[X]\right\rangle=s_{\lambda^{\prime}}\left[[n+1]_{q}\right]=\omega s_{\lambda}\left[[n+1]_{q}\right] .
$$

Since $\left\{s_{\mu}\right\}_{\mu \vdash n}$ is a basis for $\Lambda^{=n}$, it follows that for any $P \in \Lambda^{=n}$ we have that

$$
\left\langle e_{n}\left[X[n+1]_{q}\right], P[X]\right\rangle=\omega P\left[[n+1]_{q}\right] .
$$

In particular,

$$
\left\langle e_{n}\left[X[n+1]_{q}\right], h_{\mu_{1}} h_{\mu_{2}} \ldots h_{\mu_{k}}\right\rangle=\prod_{i=1}^{k} e_{\mu_{i}}\left[[n+1]_{q}\right] .
$$

Then noticing that

$$
e_{a}\left[1+q+\cdots+q^{n}\right]=q^{\left(\begin{array}{c}
a \\
2
\end{array}\right)}\left[\begin{array}{c}
n+1 \\
a
\end{array}\right]_{q}
$$

completes the proof. 


\section{Key identities}

Surprisingly, our results depend only on Propositions 1.1 and 1.2 and the following simple $q$-binomial identity. This identity can be derived from the well-known $q$-Chu-Vandermonde identity. However, the proof that follows is more illuminating.

Lemma 3.1. If $1 \leq m \leq n$ and $k \geq 0$, then

$$
\left[\begin{array}{c}
n+k \\
n
\end{array}\right]_{q}=\sum_{j=0}^{k} q^{m(k-j)}\left[\begin{array}{c}
m+j-1 \\
m-1
\end{array}\right]_{q}\left[\begin{array}{c}
n-m+k-j \\
n-m
\end{array}\right]_{q}
$$

Proof. Let $R(n, k)$ be the set of paths in the $n \times k$ rectangle from the southwest corner $(0,0)$ to the northeast corner $(n, k)$ with $k$ north edges and $n$ east edges. For such a path $\Pi$ let $\operatorname{area}(P)$ denote the area above $\Pi$. Recall that

$$
\left[\begin{array}{c}
n+k \\
k
\end{array}\right]_{q}=\sum_{\Pi \in R(n, k)} q^{\text {area( }(\Pi)} .
$$

For a fixed $m$ and for a given path $\Pi \in R(n, k)$, let $j$ be the height of the $m^{\text {th }}$ east step of $\Pi$. Note that $\Pi$ consists of a path from $(0,0)$ to $(m-1, j)$, an east step, and a path from $(m, j)$ to $(n, k)$.

Consider the example below.

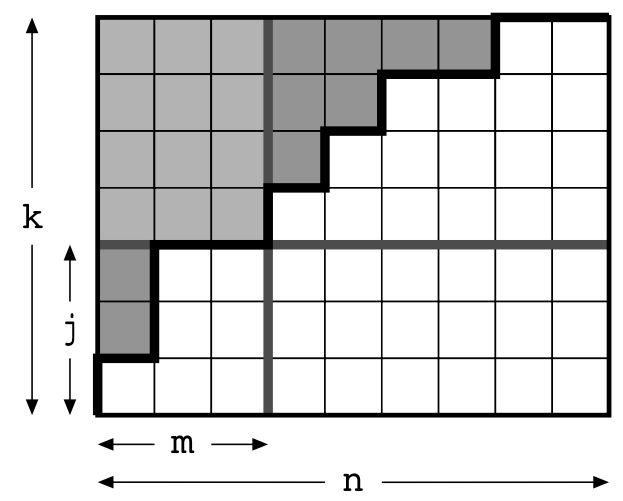

Any path of $R(m-1, j)$ and any path of $R(n-m, k-j)$ can be combined in this way to give a path $\Pi \in R(n, k)$. Conversely, any path $\Pi \in R(n, k)$ has a unique $j$ which splits the path at the $m^{\text {th }}$ east step. Hence the desired identity follows from the fact that the area of $\Pi$ is equal to $m(k-j)$ plus 
the area of the path from $(0,0)$ to $(m-1, j)$ and the area of the path from $(m, j)$ to $(n, k)$ (the three gray sections above).

For the sake of completeness, we provide sketches of the proofs of Propositions 1.1 and 1.2 in the present notation. These proofs involve similar manipulations of Parking Functions and both rely on the following well-known fact.

Let $W\left(1^{a} 2^{b}\right)$ be the set of words consisting of $a$ 1's and $b 2$ 's. Let $w_{i}$ denote the $i^{t h}$ letter of $w$. For $w \in W\left(1^{a} 2^{b}\right)$, set $\operatorname{inv}(w)=\sum_{i<j} \chi\left(w_{i}>w_{j}\right)$ and $\operatorname{coinv}(w)=\sum_{i<j} \chi\left(w_{i}<w_{j}\right)$. Then for any $n, m \geq 0$, we have that

$$
\left[\begin{array}{c}
n+m \\
m
\end{array}\right]_{q}=\sum_{w \in W\left(1^{n} 2^{m}\right)} q^{\operatorname{inv}(w)}=\sum_{w \in W\left(1^{m} 2^{n}\right)} q^{\operatorname{coinv}(w)}
$$

To deal with Parking Functions whose reading words are shuffles of $a$ small cars and $b$ big cars it is convenient to depict these functions as tableaux obtained by replacing each small car by a " 1 " and each big car by a " 2 ". This
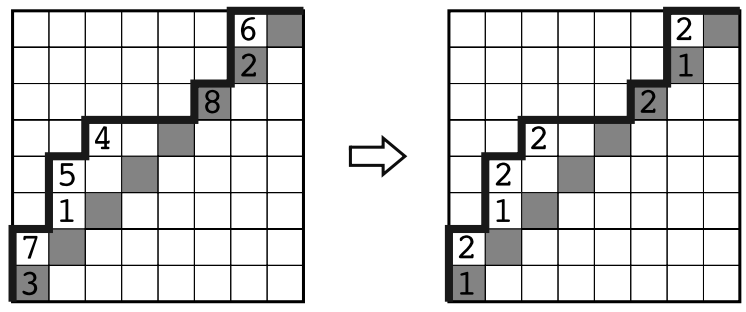

certainly does not affect the area statistic. To show that this replacement does not affect the dinv formula, we only need to point out that the shuffle condition assures that pairs of cars of the same size will never contribute a diagonal inversion. To pass from a 1,2-tableau to the original Parking Function we simply replace all the 1's successively from right to left and according to decreasing area numbers with the letters $1,2, \ldots, a$ and likewise all $2^{\prime} s$ in the same succession with the letters $a+1, a+2, \ldots, a+b$.

Let us recall that we defined

$$
\operatorname{Parkqt}_{a, b}^{(r, s)}(q, t)=\sum_{\mathrm{PF} \in \mathcal{P F}_{a, b}^{(r, s)}} t^{\operatorname{area}(\mathrm{PF})} q^{\operatorname{dinv}(\mathrm{PF})}
$$

and

$$
\operatorname{Parkqt}_{a, b}^{(s)}(q, t)=\sum_{\mathrm{PF} \in \mathcal{P F}_{a, b}^{(s)}} t^{\operatorname{area}(\mathrm{PF})} q^{\operatorname{dinv}(\mathrm{PF})}
$$


so that

$$
q^{\left(\begin{array}{c}
a+b \\
2
\end{array}\right)} \operatorname{Parkqt}_{a, b}^{(r, s)}(q, 1 / q)=\operatorname{Parkq}_{a, b}^{(r, s)}(q)
$$

and

$$
q^{\left(\begin{array}{c}
a+b \\
2
\end{array}\right)} \operatorname{Parkqt}_{a, b}^{(s)}(q, 1 / q)=\operatorname{Parkq}_{a, b}^{(s)}(q) .
$$

Propositions 1.1 and 1.2 can be obtained from the following two results by

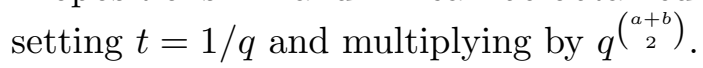

Proposition 3.1. If $0 \leq r \leq a$ and $0 \leq s<b$, then

$$
\begin{aligned}
& \operatorname{Parkqt}_{a, b}^{(r, s)}(q, t)= \\
& \quad=t^{a+b-r-s}\left[\begin{array}{c}
s+r \\
s
\end{array}\right]_{q} \sum_{h=1}^{b-s}\left[\begin{array}{c}
r+h-1 \\
h
\end{array}\right]_{q} \sum_{k=0}^{a-r} \operatorname{Parkqt}_{a-r, b-s-1}^{(k, h-1)}(q, t) .
\end{aligned}
$$

Proof. Let $0 \leq r \leq a, 0 \leq s<b, 1 \leq h \leq b-s$ and $0 \leq k \leq a-r$. Let $\mathrm{PF} \in \mathcal{P} \mathcal{F}_{a-r, b-s-1}^{(k, h-\overline{1})}$. We begin by adding a car of size 2 to the main diagonal to obtain $\mathrm{PF}^{\prime} \in \mathcal{P} \mathcal{F}_{a-r, b-s}^{(k, h)}$. We have area $(\mathrm{PF})=\operatorname{area}\left(\mathrm{PF}^{\prime}\right)$ and $\operatorname{dinv}(\mathrm{PF})=\operatorname{dinv}\left(\mathrm{PF}^{\prime}\right)$.
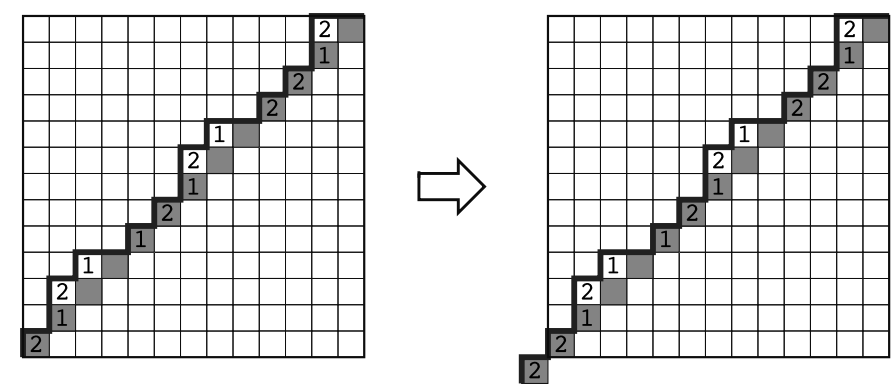

Now split up $\mathrm{PF}^{\prime}$ into $h$ blocks beginning with each car of size 2 on the main diagonal. Next we will construct an element of $\mathcal{P} \mathcal{F}_{a, b-s}^{(r, 0)}$ for each word $w$ consisting of $r$ 1's and $h$ 2's which begins with a 1 . We do this by placing each 1 we encounter within $w$ in the next available spot on the main diagonal. When we encounter a 2 in $w$, we insert the next available block of $\mathrm{PF}^{\prime}$ along the first diagonal directly on top of the 1 preceding this 2 in $w$ if there is one. Let this diagonal be known as the 1-diagonal. If there is not a 1 preceding this 2 in $w$, place the corresponding block along the 1-diagonal directly after the previous block. 

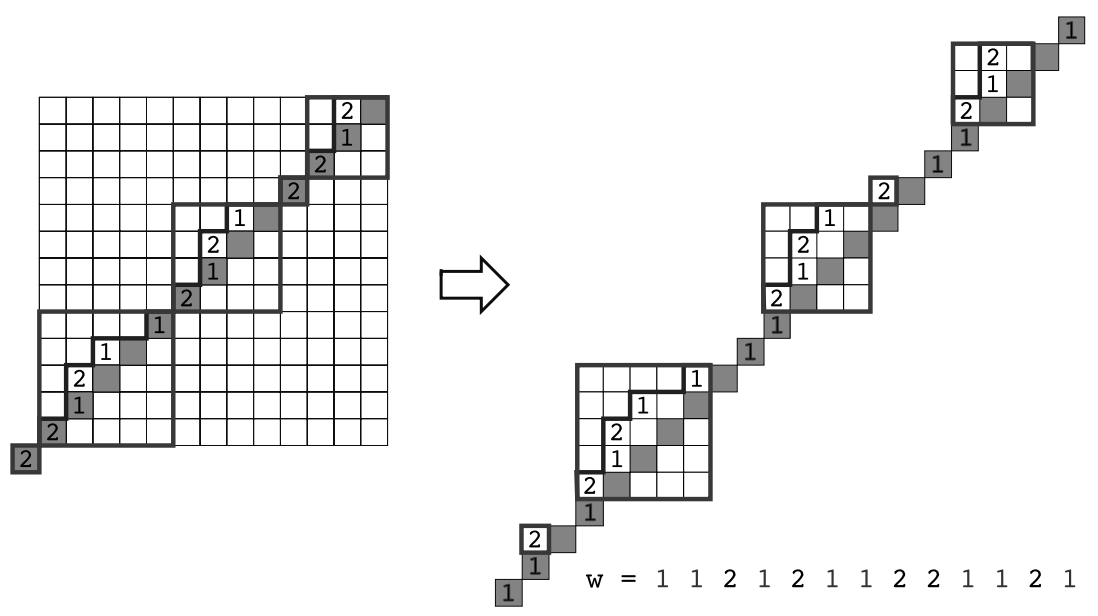

Since each of the $a+b-r-s$ cars of $\mathrm{PF}^{\prime}$ have been shifted up one diagonal and all new cars have been added to the main diagonal, the area of the resulting Parking Function is $a+b-r-s+\operatorname{area}(\mathrm{PF})$. Moreover, we have created no new primary dinv and the additional secondary dinv is equal to the number of inversions in $w, \operatorname{inv}(w)$.

Finally, we construct an element of $\mathcal{P} \mathcal{F}_{a, b}^{(r, s)}$ for each word $v$ consisting of $r$ 1's and $s$ 's. This is done by inserting 1's into the main diagonal of the previous Parking Function so that reading the main diagonal from left to right gives $v$.

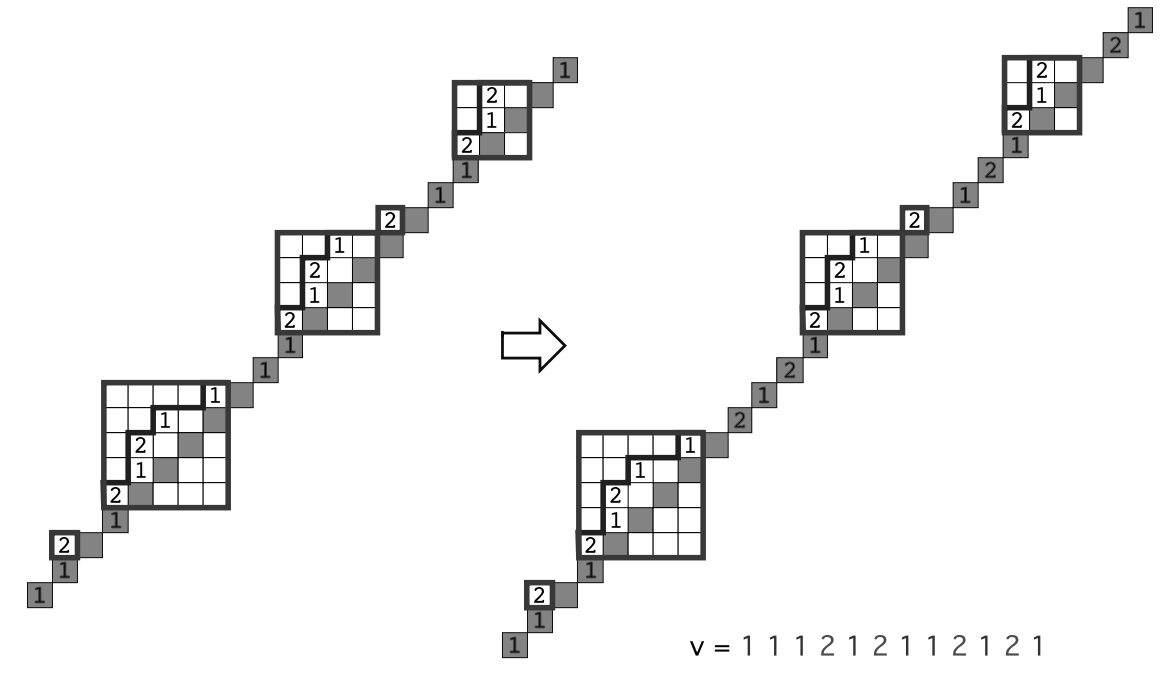


Each element of $\mathcal{P F}_{a, b}^{(r, s)}$ is uniquely created in this way by taking $k$ to be the number of 1's on the 1-diagonal, $h-1$ to be the number of 2's on the 1-diagonal, and by an appropriate choice of PF, $w$ and $v$. Therefore (3.2) follows from the observations we have made above regarding the change in area and dinv combined with (3.1).

Proposition 3.2. If $1 \leq a$ and $0 \leq s \leq b$, then

$$
\operatorname{Parkqt}_{a, b}^{(s)}(q, t)=t^{b-s} \sum_{r=1}^{a}\left[\begin{array}{c}
s+r \\
r
\end{array}\right]_{q} \operatorname{Parkqt}_{b-s, a-1}^{(r-1)}(q, t) .
$$

Proof. Let $1 \leq r \leq a$ and $0 \leq s \leq b$. Consider any $\mathrm{PF} \in \mathcal{P} \mathcal{F}_{b-s, a-1}^{(r-1)}$ and any $w \in W\left(1^{r} 2^{s}\right)$. Begin by inserting a 2 into the southwest corner of PF to get $\mathrm{PF}^{\prime} \in \mathcal{P F}_{b-s, a}^{(r)}$. As before this leaves the area and dinv unchanged.

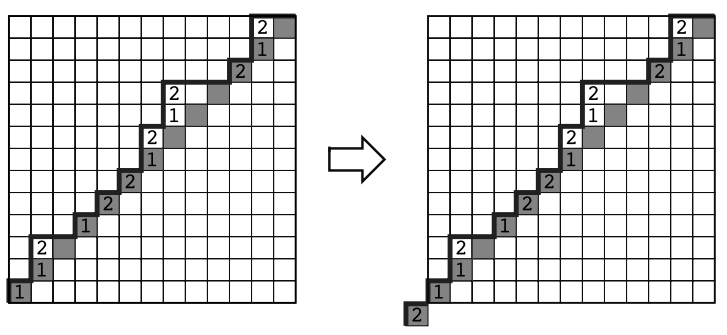

Now shift each 1 into the cell immediately west of its current position. Note that the result does not represent a Parking Function and that we have changed the supporting Dyck path. Then replace every 1 with a 2 and vice versa (i.e. turn all small cars into big cars and big cars into small cars). This gives a Parking Function $\mathrm{PF}^{\prime \prime} \in \mathcal{P} \mathcal{F}_{a, b-s}^{(0)}$. Note that area $\left(\mathrm{PF}^{\prime \prime}\right)=$ area $\left(\mathrm{PF}^{\prime}\right)+b-s$ since each 1 of $\mathrm{PF}^{\prime}$ that was shifted left increased the area.
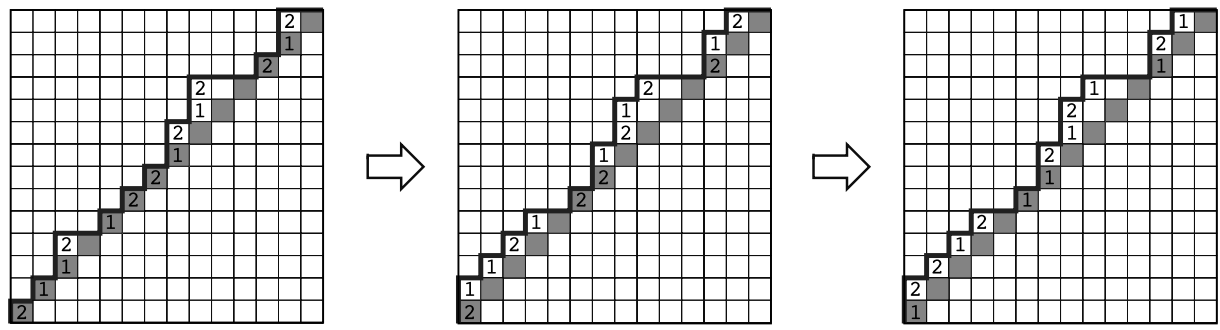

Furthermore, $\operatorname{dinv}\left(\mathrm{PF}^{\prime \prime}\right)=\operatorname{dinv}\left(\mathrm{PF}^{\prime}\right)$. This is because the pairs of 1's and 2's which cause primary dinv in $\mathrm{PF}^{\prime}$ are precisely those which cause secondary dinv in $\mathrm{PF}^{\prime \prime}$. 


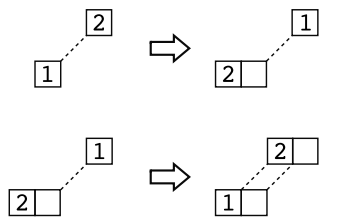

Similarly, the pairs of 1's and 2's which cause secondary dinv in $\mathrm{PF}^{\prime}$ are those which cause primary dinv in $\mathrm{PF}^{\prime \prime}$.

Now break $\mathrm{PF}^{\prime \prime}$ into $a$ blocks starting with each 1 on the main diagonal. Then insert 2's into the main diagonal according to $w$ as we did in the proof of Proposition 3.1.
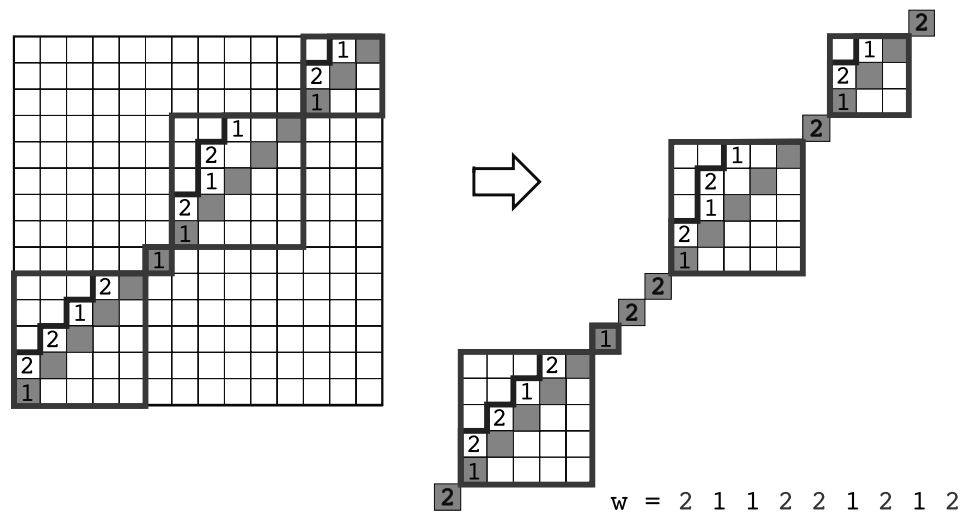

The result is a Parking Function $\mathrm{PF}^{*}$ in $\mathcal{P F}_{a, b}^{(s)}$. Clearly area $\left(\mathrm{PF}^{*}\right)=$ $\operatorname{area}\left(\mathrm{PF}^{\prime \prime}\right)=\operatorname{area}(\mathrm{PF})+b-s$. Note also that $\operatorname{dinv}\left(\mathrm{PF}^{*}\right)=\operatorname{dinv}\left(\mathrm{PF}^{\prime \prime}\right)+$ $\operatorname{coinv}(w)=\operatorname{dinv}(\mathrm{PF})+\operatorname{coinv}(w)$.

Furthermore, we can see that this operation is reversible and surjective. Hence summing over all possible choices of PF, $r$ and $w$ gives the desired result.

\section{4. $q$-binomial formulas}

In order to arrive at the formula for $\operatorname{Parkq}_{a, b}^{(r, s)}$ given in Theorem 1.1, we have the following intermediate step.

Theorem 4.1. For all $a>0$, and $0 \leq s \leq b$, we have

$$
\operatorname{Parkq}_{a, b}^{(s)}(q)=q^{\left(\begin{array}{c}
a+b \\
2
\end{array}\right)-(b-s) a}\left[\begin{array}{c}
a+b \\
a
\end{array}\right]_{q}\left[\begin{array}{c}
a+b-s-1 \\
a-1
\end{array}\right]_{q} \frac{[s+1]_{q}}{[b+1]_{q}} .
$$


When $a=0$, we have

$$
\operatorname{Parkq}_{0, b}^{(s)}(q)=q^{\left(\begin{array}{c}
b \\
2
\end{array}\right)} \chi(b=s) .
$$

Proof. When $a=0$, the only Parking Function with $a$ small cars and $b$ large cars is the one which has all large cars on the diagonal, i.e. when $b=s$. That single Parking Function has dinv $=0$ and coarea $=\left(\begin{array}{l}b \\ 2\end{array}\right)$. Hence (4.2) holds for all $0 \leq s \leq b$.

Similarly, if $b=s$ then all large cars, and consequently all small cars, are on the main diagonal. Such a Parking Function $P$ has coarea $(P)=\left(\begin{array}{c}a+b \\ 2\end{array}\right)$ and $\operatorname{dinv}(P)=\operatorname{inv}(\sigma(P))$, since all dinv occurs as primary dinv on the main diagonal. Hence

$$
\operatorname{Parkq}_{a, b}^{(b)}=q^{\left(\begin{array}{c}
a+b \\
2
\end{array}\right)}\left[\begin{array}{c}
a+b \\
a
\end{array}\right]_{q}
$$

which is the desired specialization of (4.1).

Given these two cases, we will induct on $\max \{a, b\}$. Suppose $a>0$, $b>s$ and that the claim holds for all smaller cases. Then by induction and Proposition 1.2, we have

$$
\begin{aligned}
\operatorname{Parkq}_{a, b}^{(s)}(q)= & q^{\left(\begin{array}{c}
a+b \\
2
\end{array}\right)-(b-s)-\left(\begin{array}{c}
a+b-s-1 \\
2
\end{array}\right)} \sum_{r}\left[\begin{array}{c}
s+r \\
r
\end{array}\right]_{q} \operatorname{Parkq}_{b-s, a-1}^{(r-1)}(q) \\
= & q^{\left(\begin{array}{c}
a+b \\
2
\end{array}\right)-(b-s)-\left(\begin{array}{c}
a+b-s-1 \\
2
\end{array}\right)} \sum_{r}\left[\begin{array}{c}
s+r \\
r
\end{array}\right]_{q} q^{\left(\begin{array}{c}
a+b-s-1 \\
2
\end{array}\right)-(a-r)(b-s)} \\
& \times\left[\begin{array}{c}
a+b-s-1 \\
b-s
\end{array}\right]_{q}\left[\begin{array}{c}
a-r+b-s-1 \\
b-s-1
\end{array}\right]_{q} \frac{[r]_{q}}{[a]_{q}} \\
= & q^{\left(\begin{array}{c}
a+b \\
2
\end{array}\right)-(b-s) a}\left[\begin{array}{c}
a+b-s-1 \\
a-1
\end{array}\right]_{q} \frac{[s+1]_{q}}{[a]_{q}} \\
& \times \sum_{r} q^{(b-s)(r-1)}\left[\begin{array}{c}
s+r \\
s+1
\end{array}\right]_{q}\left[\begin{array}{c}
a-r+b-s-1 \\
b-s-1
\end{array}\right]_{q} .
\end{aligned}
$$

Applying Lemma 3.1 to the sum above with $n=b+1, k=a-1$, $m=b-s$ and $j=a-r$ gives

$$
\begin{aligned}
\operatorname{Parkq}_{a, b}^{(s)}(q) & =q^{\left(\begin{array}{c}
a+b \\
2
\end{array}\right)-(b-s) a}\left[\begin{array}{c}
a+b-s-1 \\
a-1
\end{array}\right]_{q}\left[\begin{array}{c}
a+b \\
b+1
\end{array}\right]_{q} \frac{[s+1]_{q}}{[a]_{q}} \\
& =q^{\left(\begin{array}{c}
a+b \\
2
\end{array}\right)-(b-s) a}\left[\begin{array}{c}
a+b-s-1 \\
a-1
\end{array}\right]_{q}\left[\begin{array}{c}
a+b \\
a
\end{array}\right]_{q} \frac{[s+1]_{q}}{[b+1]_{q}} .
\end{aligned}
$$

By induction, the claim holds for all $0 \leq a$ and $0 \leq s \leq b$. 
Now we have the tools to prove our refinement of the Shuffle Conjecture for $k=2, t=1 / q$. Recall

Theorem 1.1. For all $0 \leq s<b$ and $0 \leq r \leq a$ we have

$$
\begin{gathered}
\operatorname{Parkq}_{a, b}^{(r, s)}(q)=q^{\left(\begin{array}{c}
a+b \\
2
\end{array}\right)-(a-r+1)(b-s)}\left[\begin{array}{c}
a+b-s-1 \\
a
\end{array}\right]_{q}\left[\begin{array}{c}
a-r+b-s \\
a-r
\end{array}\right]_{q} \\
\times\left[\begin{array}{c}
r+s \\
s
\end{array}\right]_{q} \frac{[r]_{q}}{[a-r+b-s]_{q}}
\end{gathered}
$$

and for all $0 \leq b, 0 \leq r \leq a$ we have

$$
\operatorname{Parkq}_{a, b}^{(r, b)}(q)=\chi(a=r) q^{\left(\begin{array}{c}
a+b \\
2
\end{array}\right)}\left[\begin{array}{c}
a+b \\
a
\end{array}\right]_{q} .
$$

Proof. Note that when all large cars are on the main diagonal (i.e. when $s=b$ ) all small cars must be on the diagonal as well. Hence if $r<a$, $\operatorname{Parkq}_{a, b}^{(r, b)}=0$. When $r=a$, we have that

$$
\operatorname{Parkq}_{a, b}^{(a, b)}=q^{\left(\begin{array}{c}
a+b \\
2
\end{array}\right)}\left[\begin{array}{c}
a+b \\
a
\end{array}\right]_{q}
$$

by an observation similar to that of (4.3). Note that this is not a special case of Theorem 1.1 as we assumed that $s<b$.

We will break the proof of Theorem 1.1 into two cases depending on whether $r=a$ or $r<a$.

When $r=a,(4.2)$ and Proposition 1.1 give

$$
\begin{aligned}
\operatorname{Parkq}_{a, b}^{(a, s)}(q)= & q^{(s+a)(a+b)-\left(\begin{array}{c}
s+a+1 \\
2
\end{array}\right)-1}\left[\begin{array}{c}
s+r \\
s
\end{array}\right]_{q} \\
& \times \sum_{h=1}^{b-s}\left[\begin{array}{c}
a+h-1 \\
h
\end{array}\right]_{q} \operatorname{Parkq}_{0, b-s-1}^{(h-1)}(q) \\
= & q^{(s+a)(a+b)-\left(\begin{array}{c}
s+a+1 \\
2
\end{array}\right)-1}\left[\begin{array}{c}
s+a \\
s
\end{array}\right]_{q} \\
& \times \sum_{h=1}^{b-s}\left[\begin{array}{c}
a+h-1 \\
h
\end{array}\right]_{q}^{\left(\begin{array}{c}
b-s-1 \\
2
\end{array}\right)} \chi(b-s-1=h-1) \\
= & q^{\left(\begin{array}{c}
a+b \\
2
\end{array}\right)-(b-s)}\left[\begin{array}{c}
s+a \\
s
\end{array}\right]_{q}\left[\begin{array}{c}
a+b-s-1 \\
b-s
\end{array}\right]_{q}
\end{aligned}
$$

and this is the desired specialization of Theorem 1.1. Note that the last equality is simply due to the fact that 


$$
(s+a)(a+b)-\left(\begin{array}{c}
s+a+1 \\
2
\end{array}\right)-1+\left(\begin{array}{c}
b-s-1 \\
2
\end{array}\right)=\left(\begin{array}{c}
a+b \\
2
\end{array}\right)-(b-s)
$$

which can be directly verified by expanding both sides.

Now suppose that $r<a$. Then by (4.1) and Proposition 1.1

$$
\begin{aligned}
\operatorname{Parkq}_{a, b}^{(r, s)}(q)= & q^{(s+r)(a+b)-\left(\begin{array}{c}
s+r+1 \\
2
\end{array}\right)-1}\left[\begin{array}{c}
s+r \\
s
\end{array}\right]_{q} \\
& \times \sum_{h}\left[\begin{array}{c}
r+h-1 \\
h
\end{array}\right]_{q} \operatorname{Parkq}_{a-r, b-s-1}^{(h-1)}(q) \\
= & q^{(s+r)(a+b)-\left(\begin{array}{c}
s+r+1 \\
2
\end{array}\right)-1}\left[\begin{array}{c}
s+r \\
s
\end{array}\right]_{q} \sum_{h}\left[\begin{array}{c}
r+h-1 \\
h
\end{array}\right]_{q} \\
& \times q^{\left(\begin{array}{c}
a-r+b-s-1 \\
2
\end{array}\right)-(b-s-h)(a-r)}\left[\begin{array}{c}
a-r+b-s-1 \\
a-r
\end{array}\right]_{q} \\
& \times\left[\begin{array}{c}
a-r+b-s-h-1 \\
a-r-1
\end{array}\right]_{q} \frac{[h]_{q}}{[b-s]_{q}} \\
= & q^{\left(\begin{array}{c}
a+b \\
2
\end{array}\right)-(a-r+1)(b-s)}\left[\begin{array}{c}
s+r \\
s
\end{array}\right]_{q}\left[\begin{array}{c}
a-r+b-s \\
a-r
\end{array}\right]_{q} \\
& \times \frac{[r]_{q}}{[a-r+b-s]_{q}} \sum_{h} q^{(h-1)(a-r)}\left[\begin{array}{c}
r+h-1 \\
h-1
\end{array}\right]_{q} \\
& \times\left[\begin{array}{c}
a-r+b-s-h-1 \\
a-r-1
\end{array}\right]_{q}
\end{aligned}
$$

Applying Lemma 3.1 with $n=a, k=b-s-1, m=r+1$ and $j=h-1$ gives

$$
\begin{aligned}
\operatorname{Parkq}_{a, b}^{(r, s)}(q)= & q^{\left(\begin{array}{c}
a+b \\
2
\end{array}\right)-(a-r+1)(b-s)}\left[\begin{array}{c}
s+r \\
s
\end{array}\right]_{q}\left[\begin{array}{c}
a-r+b-s \\
a-r
\end{array}\right]_{q} \\
& \times \frac{[r]_{q}}{[a-r+b-s]_{q}}\left[\begin{array}{c}
a+b-s-1 \\
a
\end{array}\right]_{q}
\end{aligned}
$$

as desired, and our proof of Theorem 1.1 is now complete.

Note that the result of summing over $r$ in the formulas of Theorem 1.1 agrees with the formulas of Theorem 4.1 by yet another application of Lemma 3.1. This computation is left for the reader. However, we cannot use these methods to find a nice closed form for $\sum_{s=0}^{b} \operatorname{Parkq}_{a, b}^{(r, s)}$. Computer 
experimentation reveals that this polynomial is not even necessarily a ratio of $q$-analogs and powers of $q$.

It remains to show that Theorem 1.1 is indeed a refinement of the Shuffle Conjecture in the case $k=2$ and $t=1 / q$. Conveniently, this can be accomplished using Lemma 3.1 one last time.

Theorem 1.2. For $a \geq 0$ and $b \geq 0$,

$$
\sum_{s} \operatorname{Parkq}_{a, b}^{(s)}=\frac{q^{\left(\begin{array}{c}
a \\
2
\end{array}\right)+\left(\begin{array}{l}
b \\
2
\end{array}\right)}}{[a+b+1]_{q}}\left[\begin{array}{c}
a+b+1 \\
a
\end{array}\right]_{q}\left[\begin{array}{c}
a+b+1 \\
b
\end{array}\right]_{q} .
$$

Proof. When $a=0$, we have already observed that the only possible Parking Function occurs when $b=s$ so that

$$
\sum_{s} \operatorname{Parkq}_{0, b}^{(s)}=q^{\left(\begin{array}{c}
b \\
2
\end{array}\right)}
$$

which is the desired specialization of (4.4).

Suppose that $a>0$ and $b \geq 0$. Then by (4.1) we have

$$
\begin{aligned}
\sum_{s} \operatorname{Parkq}_{a, b}^{(s)} & =\sum_{s} q^{\left(\begin{array}{c}
(a+b \\
2
\end{array}\right)-(b-s) a}\left[\begin{array}{c}
a+b \\
a
\end{array}\right]_{q}\left[\begin{array}{c}
a+b-s-1 \\
a-1
\end{array}\right]_{q} \frac{[s+1]_{q}}{[b+1]_{q}} \\
& =\frac{q^{\left(\begin{array}{c}
a+b \\
2
\end{array}\right)-a b}}{[a+b+1]_{q}}\left[\begin{array}{c}
a+b+1 \\
a
\end{array}\right] \sum_{s} q^{a s}\left[\begin{array}{c}
a+b-s-1 \\
a-1
\end{array}\right]_{q}\left[\begin{array}{c}
s+1 \\
1
\end{array}\right]_{q} .
\end{aligned}
$$

Now applying Lemma 3.1 with $n=a+1, m=a, k=b$ and $j=b-s$ gives

$$
\begin{aligned}
\sum_{s} \operatorname{Parkq}_{a, b}^{(s)} & =\frac{q^{\left(\begin{array}{c}
a+b \\
2
\end{array}\right)-a b}}{[a+b+1]_{q}}\left[\begin{array}{c}
a+b+1 \\
a
\end{array}\right]\left[\begin{array}{c}
a+b+1 \\
a+1
\end{array}\right]_{q} \\
& =\frac{q^{\left(\begin{array}{c}
a \\
2
\end{array}\right)+\left(\begin{array}{c}
b \\
2
\end{array}\right)}}{[a+b+1]_{q}}\left[\begin{array}{c}
a+b+1 \\
a
\end{array}\right]_{q}\left[\begin{array}{c}
a+b+1 \\
b
\end{array}\right]_{q} .
\end{aligned}
$$

which completes our proof.

In particular, this formula gives a $q$-analogue of the previously wellstudied Narayana numbers. As a special case, it gives an explicit formula 
for the specialization of a $q, t$-analogue of the Narayana numbers introduced in [5] when $t=1 / q$. Thus this paper gives a refinement and a completely combinatorial proof of a formula first calculated using symmetric symmetric function theory in [11].

Open Question: As mentioned in Section 1, this result is a refinement of an earlier algebraic formula,

$$
\operatorname{Parkqt}_{a, b+1}^{(s)}(q, t)=\left\langle\Delta_{h_{a}} E_{b+1, s}, e_{a+b+1}\right\rangle
$$

in [11] at $t=1 / q$. Different algebraic specializations of this formula, expressed using modified Hall-Littlewood polynomials, were established in [10]. It would be interesting to consider whether these new specializations had a similarly nice refinement at $t=1 / q$.

\section{References}

[1] J. C. Aval, F. Bergeron, and A. M. Garsia (2013). Combinatorics of Labelled Parallelogram Polyominoes. arXiv preprint arXiv:1301.3035.

[2] J. C. Aval, M. D'Adderio, M. Dukes, A. Hicks, and Y. Le Borgne (2013). Statistics on parallelogram polyominoes and a $q, t$-analogue of the Narayana numbers. arXiv preprint arXiv:1301.4803.

[3] F. Bergeron and A. M. Garsia (1999). Science fiction and Macdonald's polynomials. CRM Proceedings 83 Lecture Notes, American Mathematical Society 22 1-52. MR1726826

[4] F. Bergeron, A. M. Garsia, and G. Tesler (1999). Identities and positivity conjectures for some remarkable operators in the theory of symmetric functions. Methods in Appl. Anal. 6 363-420. MR1803316

[5] M. Dukes and Y. Le Borgne (2013). Parallelogram polyominoes, the sandpile model on a complete bipartite graph, and a $q, t$-Narayana polynomial. Journal of Combinatorial Theory, Series A 120 816-842. MR3022616

[6] A. M. Garsia and J. Haglund (2002). A proof of the $q, t$-Catalan positivity conjecture. Discrete Math. 256 677-717. MR1935784

[7] A. M. Garsia and M. Haiman (1996). A remarkable $q, t$-Catalan sequence and $q$-Lagrange inversion. J. Algebraic Combin. 5 191-244. MR1394305 
[8] A. M. Garsia, M. Haiman, and G. Tesler (1999). Explicit plethystic formulas for the Madonald $q, t$-Kostka coefficients. Séminaire Lotharingien de Combinatoire $\mathbf{B} 4 \mathbf{2 m} 45 \mathrm{pp}$.

[9] A. Garsia, A. Hicks, and A. Stout (2011). The case $k=2$ of the Shuffle Conjecture. J. Comb. 2 193-229. MR2913193

[10] A. M. Garsia, G. Xin, and M. Zabrocki (2013). A three shuffle case of the compositional parking function conjecture. arXiv preprint arXiv:1208.5796.

[11] J. Haglund (2004). A proof of the q,t-Schröder conjecture. Internat. Math. Res. Notices 11 525-560. MR2038776

[12] J. Haglund, M. Haiman, N. Loehr, J. B. Remmel, and A. Ulyanov (2005). A combinatorial formula for the character of the diagonal coinvariants. Duke J. Math. 126 195-232. MR2115257

[13] A. G. Konheim and B. Weiss (1966). An occupancy discipline and applications. SIAM J. Applied Math. 14 1266-1274.

[14] I. G. Macdonald (1995). Symmetric Functions and Hall Polynomials, 2 ed. Oxford Mathematical Monographs, New York. MR1354144

ANGELA Hicks

Department of Mathematics, Building 380

STANFORD, CALIFORNIA 94305

USA

E-mail address: ashicks@stanford.edu

EMILY LEVEN

9500 Gilman Drive \# 0112

La Jolla, CALifornia 92093-0112

USA

E-mail address: esergel@ucsd.edu

ReCEIVEd June 25, 2013 